\title{
EQUIVOCATIONS FOR HOMOPHONIC CIPHERS
}

\author{
Andrea Sgarro \\ Istituto di Matematica \\ università di Trieste \\ 34100 Trieste (Italy)
}

Abstract. Substitution ciphers can be quite weak when the probability distribution of the message letters is distinctly non-uniform. A timehonoured solution to remove this weakness is to "split" each high-pro bability letter into a number of "homophones" and use a substitution cipher for the resulting extended alphabet. Here the performance of a homophonic cipher is studied from a shannon-theoretic point of view. The key and message equivocations (conditional entropies given the in tercepted cryptogram) are computed both for finite-length messages and "very long" messages. The results obtained are strictly related to those found by Blom and Dunham for substitution ciphers. The key space of a homophonic cipher is specified carefully, so as to avoid misunder standings which appear to have occurred on this subject.

Work done within the research program of GNIM-CNR. 
Simple substitution ciphers (s.s.c.'s) are probably the oldest type of ciphers put to work, and yet they are still in good health in the form of (individually weak) components of (hopefully good) complex cipher system (e.g. the Data Encryption standard). The key of a s.s.c. is a permutation of the message-letter alphabet $A=\left\{a_{1}, a_{2}, \ldots, a_{5}\right\}, s \geqq 2$; once a key is chosen each single letter output by the message source is replaced by its substitute. S.s.c.'s have been studied rather deeply in the last decade; $c f . / 1,2,3 /$; in the first two papers the strength of a cipher is assessed by evaluating the equivocations ("uncertainties") on side of the spy who has intercepted a cryptogram (key equivocation or message equivocation, according whether the spy is interested in finding out the correct key or the correct message); in /3/ the error probabilities are evaluated when the spy uses the best statistical pro cedure to recover the correct key or message from the intercepted cryp togram. Further work on s.s.c.'s is done in /4/, which contains a discus sion on the role of the "Shannon-theoretic approach" to cryptography and, more generally, on the relevance of purely statistical cryptogra phic models.

A s.s.c. is very weak when the probability distribution (p.d.) P ruling the message source, which we assume to be memoryless and station ary, is distinctly non-uniform; $\left(\mathrm{p}=\left\{\mathrm{p}_{1}, \mathrm{p}_{2}, \ldots, \mathrm{p}_{\mathrm{s}}\right\}, \mathrm{p}_{i}>0,\left[\mathrm{p}_{i}=1\right.\right.$; un specified summations are meant over all values of the index). A timehonoured solution to remove this weakness is to make use of a cryptogramletter alphabet $C$ of size $t$ larger than $s$, the size of the message-let ter alphabet; for example, the letters of $C$ might be the ordered couples of message letters. Then any large probability $p_{i}$ can be broken down by associating to the corresponding letter $a_{i}$ many possible cryptogram substitutes, $t_{i}$, say: each time $a_{i}$ occurs in the message one of these is chosen at rancon and actually substituted for $a_{i}$. The resulting $c \underline{i}$ pher is called a Eomophonic cipher (or, rather, a simple, that is singleletter, homophonic cipher; a more formal description is given below). Homophonic ciphers, which are a generalization of s.s.c.'s (refound for $t_{i}=1,1 \leqq i \leqq s$, that is, essentially, when $A=C$, have been recently 
studied in /5/. In this paper we take the equivocation approach to assess the strength of homophonic ciphers, thereby generalizing the work done in $/ 1 /$ and $/ 2 /$ for s.s.c.'s.

A mathematical tool which we shall use is the notion of an exact type. Consider $A^{\mathrm{n}}$, the set of the $\mathrm{s}^{\mathrm{n}}$ sequences of length $\mathrm{n}$ built over $A$; an exact type (of order $n$ over $A$ ) is a subset of $A^{n}$ made up of a sequence together with all its permutations. Of course, if $\left(n_{1}, n_{2}, \ldots, n_{s}\right)$ is the composition of any of these sequences, $n_{i}$ being the number of occur rences of letter $a_{i},\left(n_{i} \geq 0,\left[n_{i}=n\right)\right.$, the size of the corresponding exact type is the multinomial coefficient $n ! / n_{1} ! n_{2} ! \ldots n_{s} !$. Statisticians will recognize here an obvious link with the notion of sufficient statistic; we simply stress that the sequences of an exact type have all the same probability. A powerful technique based on asymptotically tight bounds for the size and the probability of exact types has been made popular in the circle of information theorists by the fundamental textbook $/ 6 /$. This technique is applied in /3/ to the error probability approach to s.s.c.'s and in/7/ to the equivocation approach to the same ciphers.

Before going to mathematical developments, we have to give a more formal description of a homophonic cipher. Two alphabets, $A$ and $C$ $=\left\{c_{1}, c_{2}, \ldots, c_{t}\right\}, t \geqq s$, are given, $c$ being the cryptogram-letter alpha bet; also $s$ integers are given which sum to $t: t_{1}, t_{2}, \ldots, t_{s}, t_{i} \geqq 1$, $\sum t_{i}=t$. A key is specified by giving $s$ disjoint subsets of $c$ of size $t_{1}, t_{2}, \ldots$ and $t_{s}$, respectively. Each time letter $a_{i}$ is output by the message source, one of the $t_{i}$ letters of the $i-t h$ subset is chosen with (conditional) probability $1 / t_{i}$ and is substituted for letter $a_{i}$. The knowledge of the key is enough to reconstruct the correct message from any of the possible corresponding cryptograms. Before transmission begins, a key is chosen at random and independently of the message output by the source; the key is communicated to the legitimate receiver via a secure special channel; ("at random" means that the key is a uniform random variable, or $\mathrm{r.v}$, over the set of all possible keys). The cryptogram is cerived from the message and sent over the normal unsafe channel, where it is intercepted by the spy.

We find it convenient to give a more careful description of the key of a homophonic cipher. Such a key can be represented by a sequence in 
$A^{t}$ with composition $\left(t_{1}, t_{2}, \ldots, t_{s}\right)$ (alphabet letter $a_{i}$ occurs $t_{i}$ times, $\left.\sum t_{i}=t\right)$. The meaning of this representation is that if $a_{i}$ is the $j-t h$ component of the sequence, then $c_{j}$ is a possible substitute for $a_{i}$ under the given key. We shall actually identify each possible key with the corresponding sequence in $A^{t}$, so that for us the set of keys will be an exact type in $A^{t}$. Clearly the number of all keys for a homo phonic cipher $\left(A, C ; t_{1}, t_{2}, \ldots, t_{s}\right)$ is the multinomial coefficient $t ! / t_{1} ! t_{2} ! \ldots t_{s} !$

As shown in $/ 5 /$, a homophonic cipher induces a s.s.c. in a quite natural way. A presentation follows which suits our purposes. Take an extended alphabet $U=\left\{u_{1}, u_{2}, \ldots, u_{t}\right\}$ with the same size $t$ as the crypto gram alphabet $C$; although it would not be restrictive to take $U=C$ we keep them separate for the sake of notational clarity. The elements of $U$ will be denoted at places by symbols like $a_{i j}, 1 \leqq i \leqq s, 1 \leqq j \leqq t_{i}$; in other words in $U$ each message letter $a_{i}$ is duplicated $t_{i}$ times: the letters $a_{i j}$ are called the homophones of letter $a_{i}$. No ambiguity should result from the fact that the letters of $U$ have two names, e.g. $u_{1}$ is also called $a_{i j}$ for some $i$ and some $j$. A dumy memoryless and stationary source with alphabet $U$, called the extended source, is now built in the following way: each time the message source outputs a letter $a_{i}, 1 \leq i \leq s$, the extended source outputs a letter $a_{i j}, 1 \leqq j \leqq t_{i}$, with (conditional) uniform probability; then the (absolute) probability of letter $a_{i j}$ is $p_{i} / t_{i}$. We call $p^{*}$ the p.d. made up of these probabilities; $p^{*}$ rules the statistical behaviour of the extended source. Note also that the output of the message source is a deterministic function of the simultaneous output of the extended source.

Take now the s.s.c. $(U, C)$, whose $t$ ! keys can be represented (cf. above) as sequences in $U^{t}$ where each letter occurs exactly once. To any key for $(U, C)$ we can associate a key for $\left(A, C ; t_{1}, t_{2}, \ldots, t_{s}\right)$ replacing each $a_{i j}$ in the $U^{t}$ sequence by $a_{i}$. A homophonic cipher can be put to work in the following way, which is readily shown to be equivalent to the original description. A key is chosen for the s.s.c. $(U, C)$. The message source is set going together with the extended source syncronized with it. The key is applied to the extender message to give the cryptogram. From this key a "short" key can be obtained as above to be communicated 
to the legitimate receiver. The "short" key applied to the cryptogram does not allow the legitimate receiver to recover the extended message, but it does allow him to recover the original message over $A$, which is what he needs to know. The key for the s.s.c. $(U, C)$ is a uniform $r . v$. $K^{*}$ with $t$ ! values, while the "short" key for the homophonic cipher is a uniform $r . v$. $K$ with $t ! / t_{1} ! t_{2} ! \ldots t_{s}$ ! values. We shall also write

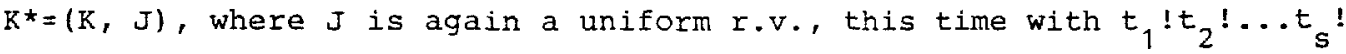
values, which identifies $K^{*}$ once $K$ is known; note that $K$ and $J$ are independent, as it appears from the values of the respective probabili ties. $K, J$ and $K^{*}$ will be referred to as the actual key, the supplemen tary key and the extended key. We stress the distinction between $k$ an $\bar{d}$ $K^{*}$ : it is the former which is the "true" key of the homophonic cipher, while $K^{*}$ contains the "redundant information" $J$; $c f$. the discussion at the end of section 4 .

Let the r.v.'s $M_{n}, U_{n}$ and $C_{n}$ denote the first $n$ letters output by the message source, the extended source and the cryptogram source, respectively. Some relations for relevant entropies are already im plicit from the foregoing: $H\left(M_{n} \mid U_{n}\right)=0, H\left(M_{n} \mid K, C_{n}\right)=0, H\left(M_{n} \mid K\right)=H\left(M_{n} \mid K^{*}\right)=$ $=H\left(M_{n}\right), H(K, J)=H(K)+H(J)$, etc. In the following section we shall assess the performance of a homophonic cipher by evaluating its equivocations.

\section{The equivocations.}

The equivocations of interest are: $H\left(k \mid C_{n}\right)$, the key equivocation, $H\left(K \mid M_{n}, C_{n}\right)$, the key appearance equivocation, interesting in the case of "chosen plain-text attacks", and, most important of the three, $H\left(M_{n} \mid C_{n}\right)$, the message equivocation. Since $(U, C)$ is a s.s.c. we already know a lot about its own equivocations, $H\left(K^{*} \mid C_{n}\right), H\left(K^{\star} \mid U_{n}, C_{n}\right)$ and $\mathrm{H}\left(\mathrm{U}_{\mathrm{n}} \mid \mathrm{C}_{\mathrm{n}}\right)$; cf. $/ 1,2,7 /$. Only the first will be needed. Its value is

$$
H\left(K * \mid C_{n}\right)=\log A+\sum_{r} P *^{n}(T) \log \frac{\sum_{Q} Q^{n}\left(\underline{u}_{r}\right)}{P^{*}{ }^{n}\left(\underline{u}_{r}\right)}
$$

where $A=A\left(P, t_{1}, t_{2}, \ldots, t_{s}\right)=d_{1} ! d_{2} ! \ldots d_{h} !, h$ being the number of distinct probabilities appearing in $\mathrm{P}^{*}$, the first $\mathrm{d}_{1}$ times, the second $\mathrm{d}_{2}$ times, 
etc.; $d_{1}+d_{2}+\ldots+d_{h}=t$; the $r$-summation is extended over all exact types $T_{r}$ in $U^{n} ; \underline{u}_{r}$ is any sequence in $T_{r}$; the $Q$-summation is extended over all p.d.'s $Q$ which are obtained by permuting the components of $P^{*}$, including $P^{*}$ itself: these p.d.'s are only $t$ !/A owing to ties in the components of $P^{*}$; of course $Q^{n}$ is the memoryless extension of $Q$ over $U^{n}$. The term $\log \mathrm{A}$ is a constant; it is certainly non-zero for a strict IY homophonic cipher (one for which $t \geqq s+1$ ). The second term goes to zero and it is exactly zero when $P^{*}$ is uniform and $A$ achieves its maximum value log $t$ ! (cf. also section 3 ).

Some simple identities are helpful. For example: $H\left(K^{*} \mid C_{n}\right)=H\left(K, J \mid C_{n}\right)=$ $=H\left(K \mid C_{n}\right)+H\left(J \mid K, C_{n}\right)$; as $H\left(K^{*} \mid C_{n}\right)$ is known, it will be enough to compute $\mathrm{H}\left(\mathrm{J} \mid \mathrm{K}, \mathrm{C}_{\mathrm{n}}\right)$ and then use:

$$
H\left(K \mid C_{n}\right)=H\left(K * \mid C_{n}\right)-H\left(J \mid K, C_{n}\right)
$$

Further: $H\left(K, M_{n} \mid C_{n}\right)=H\left(K \mid C_{n}\right)+H\left(M_{n} \mid K, C_{n}\right)=H\left(K \mid C_{n}\right)$ because $M_{n}$ is a deter ministic function of key and cryptogram; and also $H\left(K, M_{n} \mid C_{n}\right)=H\left(M_{n} \mid C_{n}\right)+$ $+H\left(K \mid M_{n}, C_{n}\right)$. By comparision (cf. $\left./ 2 /\right)$ :

$$
H\left(M_{n} \mid C_{n}\right)=H\left(K \mid C_{n}\right)-H\left(K \mid M_{n}, C_{n}\right)
$$

Now we deal directly with $H\left(J \mid K, C_{n}\right)$ and $H\left(K \mid M_{n}, C_{n}\right)$

Theorem 1.

$$
H\left(J \mid K, C_{n}\right)=H(J)=\sum \log t_{i} !
$$

Proof. Assume $(k, j)$ and $(k, i)$ are two extended keys for the s.s.c. $(U, C)$ with the same actual key $k$. With respect to each other these keys only scramble equiprobable homophones relative to the same messagealphabet letter. Therefore, for any cryptogram $\underline{c}$, Prob $\left\{c_{n}=\underline{c} \mid K^{*}=(k, j)\right\}=$ Prob $\left\{C_{n}=\underline{c} \mid K^{*}=(k, i)\right\}_{j}^{l}$. This means that $C_{n}$ and $J$ are conditionally indepen dent given $K$, and therefore $H\left(J \mid K, C_{n}\right)=H(J \mid K)$. But we already know that $J$ and $K$ are independent, so $H(J \mid K)=H(J)$. To complete the theorem, recall that $J$ is a uniform $r . v$. with $t_{1} ! t_{2} ! \ldots t_{s} !$ values $Q E D$

In theorem 2 the $r$-summation is extended over all exact types $T_{r}$ in $U^{\mathrm{n}}$ and $h_{i}=h_{i}\left(T_{r}\right)$ is the number of distinct letters $a_{i j}$ which do not occur in the sequences of $T_{r}, 1 \leqq i \leqq s, 0 \leqq h_{i} \leqq t_{i},\left[h_{i} \leqq t-1\right.$.

Theorem 2.

$$
H\left(K \mid M_{n}, C_{n}\right)=\sum P^{*}(T r) \quad\left[\log \left(\sum h_{i}\right) !-\sum \log h_{i} !\right]
$$

The non-zero terms in the summation are those for which at least two $h_{i}$ 's are positive, that is, at least two unequivalent homophones are 
missing.

Proof. Assume that a couple message-cryptogram, 뜨, $\underline{c}$, is given of pos itive joint probability. Let us try to reconstruct the key, which is a sequence in $A^{t}: e . g$. . if $a_{i}$ and $c_{i}$ are letters in the same position in the given couple, $a_{i}$ is the $j-t h$ component of the key. However, gaps might be left because letter $a_{i}$ might not occur, or it might occur in correspondence to less than $t_{i}$ distinct cryptogram letters. If $h_{i}$ denotes the number of times letter $a_{i}$ is missing in the partially reconstructed key, the number of possible keys left is $\left(\left[h_{i}\right) ! / h_{1} ! h_{2} ! \ldots h_{s} !\right.$. 9ecause of symmetry each such key has the same conditional probability, and so

$$
H\left(K \mid M_{n}=m, C_{n}=c\right)=\log \left(\sum h_{i}\right) !-\sum \log h_{i} !
$$

Note that the integers $h_{i}$ can be computed directly from the extended se quence $u$ output by the extended source, $h_{i}$ being simply the number of distinct letters $a_{i j}$ which do not occur in $\underline{u}$. Note also that the set of u-sequences with given integers $h_{i}$ is a union of exact types. There fore, grouping together $\underline{u}$-sequences in the same type:

$$
H\left(K \mid M_{n}, C_{n}\right)=\sum P^{*}\left(T_{r}\right)\left[\log \left(\sum h_{i}\right) !-\left[\log h_{i} !\right]\right.
$$

Clearly the quantity inside square brackets is zero only when at most one $\mathrm{h}_{i}$ is positive. This proves the last statement in the theorem. QED Note that the key-appearance equivocation is zero only for $t=2$, and then the homophonic cipher is also a s.s.c. $(s=t=2)$.

Now (1), (2) and (3), together with the two theorems, give the exact values of the equivocations $H\left(K \mid C_{n}\right), H\left(K \mid M_{n}, C_{n}\right)$ and $H\left(M_{n} \mid C_{n}\right)$.

\section{Asymptotic results.}

It will be shown now that the key-appearance equivocation $H\left(K / M_{n}, C_{n}\right)$ becomes negligible with increasing message length $n$. Therefore for large $n^{\prime} s$ both $H\left(K / C_{n}\right)$ and $H\left(M_{n} \mid C_{n}\right)$ are approximately equal to the constart term

$$
\log A-\sum \log t_{i} \text { ! }
$$

("unremovable uncertainty"); cf. also the observations below formula (1). Note that the factors $a_{j}$ which appear in the definition of $A$ (cf. again (1)) are made up summing one or more $t_{i}$ 's bacause equivalent 
homophones have all the same probability. So, as it should be, the unremovable uncertainty is non-negative. It is zero when only equiva lent homophones are equiprobable (the numbers $d_{j}$ and the numbers $t_{i}$ are the same up to their order).

Now we investigate the behaviour of $H\left(K / M_{n}, C_{n}\right)$ as a function of $n$. To extend the validity of theorem 3 below to the case $t=2$, when $H\left(K \mid M_{n}, C_{n}\right)$ is zero, we adopt the (natural) convention that a term of the form $\exp \left\{n \sum_{-}^{-}+\varepsilon_{n}\right\}$, $\lim _{\mathrm{n}} \varepsilon_{\mathrm{n}}=0$, means zero. We set $D=D\left(P, t_{1}, t_{2}, \ldots, t_{s}\right)={ }_{1 \leqq 1} \operatorname{mig}_{S s}\left(P_{i} / t_{i}+p_{f} / t_{f}\right) \leqq 1 \quad(D=1$ if and only if $s=t=2)$. Theorem 3 .

$$
H\left(K \mid M_{n}, C_{n}\right)=\exp \left\{n\left[\log (1-D)+\varepsilon_{n}\right\}\right\}, \lim \varepsilon_{n}=0
$$

Proof. Take $t \geq 3$. We start with the obvious bounds:

$$
\log 2 \sum_{r} P^{*}\left(T_{r}\right) \leqq H\left(K \mid M_{n}, C_{n}\right) \leqq \log (t-1) ! \sum_{r} P^{n}\left(T_{r}\right)
$$

the summations being restricted to types which correspond to non-zero terms in the summation of theorem 2 . Denote by $M(i, j ; f, g)$ the set of $U^{n}$-sequences such that $a_{i j}$ and $a_{f g}$ are missing in them; $1 \leqq i<f \leqq s$, $1 \leqq j \leqq t_{i}, 1 \leqq g \leqq t_{j} ; M(i, j ; f, g)$ is a union of exact types. One has:

$$
\sum_{\underline{r}} \mathrm{P}^{\mathrm{n}}\left(T{ }_{r}\right)=P *^{\mathrm{n}}\left(\cup_{\mathrm{r}}{ }^{T}{ }_{r}\right)=P \star^{\mathrm{n}}(U M(i, j ; f, g)) \text {; }
$$

the sets in the latter union, which is not disjoint, are no more than $\left(\frac{5}{2}\right)[(t-1) !]^{2}$. One has also:

$$
p^{*^{-}}(M(i, 1 ; f, 1))=p^{*^{n}}(M(i, j ; f, g))=\left(1-p_{i 1}^{*}-p_{f 1}^{\star}\right)^{n}=\left(1-p_{i} / t_{i}-p_{f} / t_{f}\right)^{n}
$$

Assume that $D$ is achieved, say, for $i=1, f=2$. Then:

$$
\mathrm{P} *^{\mathrm{n}}\left(M(1,1 ; 2,1)=(1-D)^{\mathrm{n}} \geq \mathrm{P} *^{\mathrm{n}}(M(i, j ; f, g))\right.
$$

and the bounds for $\mathrm{H}\left(\mathrm{K} \mid \mathrm{M}_{\mathrm{n}}, \mathrm{C}_{\mathrm{n}}\right)$ can be relaxed to:

$$
\log 2(1-D)^{n} \leq H\left(K \mid M_{n}, C_{n}\right) \leqq\left(\frac{s}{2}\right)\left\lceil\left.(t-1) !\right|^{2} \log (t-1) !(1-D)^{n}\right.
$$

This ends the proof. QED

Observe that the proof of the theorem implicitly gives asymptotically tight bounds for $\varepsilon_{n}$ which are independent of $P(t \geq 3)$ :

$$
n^{-1} \log \log 2 \leqq \varepsilon_{n} \leqq n^{-1} \log \left\{\left(\frac{s}{2}\right)[(t-1) !]^{2} \log (t-1) !\right\}
$$

The parameter $D$ which appears in theorem 3 does not coincide with the corresponding parameter obtained by Dunham /2/ for the key appear ance equivocation $H\left(K * \mid U_{n}, C_{n}\right)$ of the s.s.c. $(U, C)$. He proved that $H\left(K * \mid U_{n}, C_{n}\right)=\exp \left\{n\left[\log (i-\hat{D})+\hat{E}_{n}\right]_{1}^{l}, \lim _{n} \hat{\varepsilon}_{n}=0\right.$, where $\hat{D}$ is the sum of the two smallest components of $\mathrm{P}^{*}$; since these components may be relative to equivalent homophones, one has $\hat{\mathrm{D}} \leq \mathrm{D}$. 
The asymptotic behaviour of $\mathrm{H}\left(\mathrm{K}^{*} \mid \mathrm{C}_{\mathrm{n}}\right)$ is well-known $(\mathrm{cf}, 11,7 /)$, and so we have all we need. We shall write down explicitly the asym ptotic formula for $\mathrm{B}\left(\mathrm{M}_{n} \mid \mathrm{C}_{n}\right)$, the most complex (and in a way the most relevant) of the three equivocations:

$$
\begin{aligned}
& H\left(M_{n} \mid C_{n}\right)=H\left(K \mid C_{n}\right)-H\left(K \mid M_{n}, C_{n}\right)=\log A-\sum \log t_{i} ! \\
& \left.+\exp \left\{n \mid \log (1-B)+\delta_{n}\right]\right\}-\exp \left\{n\left[\log (1-D)+\varepsilon_{n-1}\right], \lim \delta_{n}=\lim \varepsilon_{n}=0\right.
\end{aligned}
$$

$B=B\left(P, t_{1}, t_{2}, \ldots, t_{s}\right)$ is defined as min $\left(\sqrt{p_{i} / t_{i}}-\sqrt{p_{j} / t_{j}}\right)$, the minimum being taken over all distinct $P^{*}$-probabilities, $1 \leq i, j \leqq s, p_{i} / t_{i} \neq p_{j} / t_{j}$; $B$ is set equal to 1 for $P$ * uniform, so that the corresponding expo nential term becomes zero. of course, if $B<D$, one can write the message equivocation as:

$$
H\left(M_{n} \mid C_{n}\right)=\log A-\left[\log t_{i} !+\exp \left\{n \left[\log (1-B)+\delta_{n}^{-} \mid l, \lim \delta_{n}^{\prime}=0,\right.\right.\right.
$$

while, if $B>D$, one has instead:

$$
H\left(M_{n} \mid C_{n}\right)=\log A-\left[\log t_{i} !-\exp \left\{n \left[\log (1-D)+\varepsilon_{n}^{-} \mid l, \lim _{n} \varepsilon_{n}^{\prime}=0 .\right.\right.\right.
$$

\section{Final remarks.}

At the beginning of section 3 it has already been pointed out that, for large message lengths $\mathrm{n}$, both the key and the message equivocation are approximately equal to the "unremovable uncertainty" $\log A-\sum \log t_{i} ! \geq 0$. The condition for the unremovable uncertainty to be zero is that only equivalent homophones are equiprobable. An advantageous situation is found instead when $P^{*}$ is uniform (all the homophones are equiprobable); then the homophonic cipher is said to be matched $(c f . / 5 /)$ and the un removable uncertainty equals $\log t !-\left[\log \left(t p_{i}\right) !, t p_{i}\right.$ integers. In prin ciple, when the components of $P$, the p.d. of the message source, are rational, one can always achieve $P$ * uniform for a sufficiently large cryptogram-alphabet size $t$; however, alphabet extension runs counter to complexity requirements (it also leads to the growth of the term $\left[\log t_{i} !\right)$. Once a threshold $T>s$ is given, always assuming that the cipher will be used for a long time, one should judiciously choose the parameters $t, t_{1}, t_{2}, \ldots, t_{s}, s \leqq t \leqq T$, in order to achieve a large un removable uncertainty. Were it not so, the performances of the homophonic cipher might even be worse than those of the s.s.c. (A, A) for the same 
message source, for example when equal probabilities $p_{i}$ and $p_{j}$ are split to give distinct probabilities $p_{i} / t_{i}$ and $p_{j} / t_{j}$, so that letters $a_{i}$ and $a_{j}$ become statistically distinguishable only in the case of the homo phonic cipher.

Assume a cipher system $\left(K, M_{n}, C_{p}\right)$ is given. We call two keys $k$ and $h$ indistinguishable when $\mathrm{T}_{\mathrm{k}}^{-1}(\underline{c})=\mathrm{T}_{\mathrm{h}}^{-1}(\underline{c})$ for all cryptograms $\underline{c}$, of any length $\left(\mathrm{T}_{\mathrm{k}}^{-1}(\cdot)\right.$ denotes the cryptogram-to-message transformation deter mined by key $k$; note that in the case of a homophonic cipher the mes sage-to-cryptogram transformation $\mathrm{T}_{\mathrm{k}}(\cdot)$ is not deterministically de fined). The spy (and also the authorized receiver, for that) is inter ested only in the equivalence class of indistinguishable keys to which $k$ belongs, rather than in $k$ itself. Sometimes the extended key, $k^{*}$, has been misinterpreted as the "true" key of a (strictly) homophonic cipher. If one neglects the fact that distinct extended keys with the same actual key are indistinguishable, one is lead to give over-optimistic evaluations of the cipher's performances. In particular, the negative term $-\left[\log t_{i} !\right.$, which appears both in key and message equivocation, is ignored. Our description of the various types of "keys" in terms of suitable exact types makes it transparent why the "true" key of a homo phonic cipher is precisely the actual key, $k$. Distinct actual keys are always distinguishable.

\section{References.}

/1/ R.J. Blom, "Bounds on key equivocation for simple substitution ciphers",IEEE Trans. Inform. Theory, vol. IT-25, pp. 8-18, Jan 1979.

/2/ J.G. Dunham, "zounds on message equivocation for simple substitu tion ciphers", IEEE Trans. Inform. Theory, vol. IT-26, pp. 522-527, Sept. 1980

13/ A. Sgarro, "Error probabilities for simple substitution ciphers", IEEE Trans. Inform. Theory, vol. IT-29, pp. 190-198, March 1983

/4/ A. Sgarro, "Exponential-type parameters and substitution ciphers"; a preliminary version called "Remarks on substitution ciphers" has been presented at the 1983 IEEE ISIT held at St. Jovite (quebec) 
/5/ J.G. Dunham, "Substitution and transposition ciphers", submitted /6/ I. Csiszar and J. Körner, Information theory: Coding theorems for discrete memoryless systems. New York: Academic, 1981

/7/ A. Sgarro, "Simple substitution ciphers", in secure digital commu nications, ed. by G. Longo. CISM Courses and Lectures No. 279, Springer-Verlag, Wien-New York, pp. 61-77, 1983 